\title{
A CROSS-PLATFORM NUMERICAL MODEL OF ION-WALL COLLISIONS*
}

\author{
S. Veitzer ${ }^{\#}$, P. Stoltz, Tech-X Corporation, Boulder, CO, U.S.A. \\ J.-L. Vay, LBNL, Berkeley, CA, U.S.A. \\ A. W. Molvik, R. Cohen, LLNL, Livermore, CA, U.S.A.
}

\begin{abstract}
Ion collisions with beam-pipe walls are a significant source of secondary electron clouds and desorbed neutral gasses in particle accelerators. Ions may reflect from beam-pipe walls and undergo further collisions downstream. These effects can cause beam degradation and are expected to be problematic in the design of heavy ion accelerators. The well-known SRIM code provides physically based Monte Carlo simulations of ion-wall collisions. However, it is difficult to interface SRIM with high-performance simulation codes. We present details on the development of a package of Python modules that integrate the simulation of ion-wall interactions at grazing incidences with the high-performance particle-in-cell and electron cloud codes WARP and POSINST. We have a Python-based set of routines that calculate reflected angles and energies of ions that strike beam-pipe walls. The results are based on interpolation of benchmark simulations run in SRIM for a variety of relevant incident angles and energies.
\end{abstract}

\section{MOTIVATION AND APPROACH}

Ion-wall interactions are the source of secondary electrons and desorbed gas in accelerator beam pipes. These electrons can cause beam instability through electron cloud effect, and the neutral gas can cause vacuum pressure rise and, through ionization, more electron cloud. To understand how to mitigate ion-wall interactions, one would like to use simulation, however the best known code in this field, SRIM [1], runs only on the Windows platform and is too slow for interfacing with other codes in real time.

As an example of how researchers would like to use ion-wall interactions in simulation, we consider simulations of the High Current Experiment (HCX) [2] at Lawrence Berkeley National Laboratory. The HCX looks at transport of high-current $1.0 \mathrm{MeV}$ potassium ions. Simulations of the HCX use the WARP code to track the electro-magnetic interactions of the ions and the accelerator. When an ion hits the wall in the simulation, one would like to interface with a code like SRIM to find out how that ion behaves (does it scatter, penetrate, produce secondaries, etc). However, WARP typically runs in a Linux environment, and SRIM does not. Furthermore, using SRIM directly takes too much time and would slow down WARP too much for practical use.

Our approach to both make ion-wall simulations crossplatform and fast is to reduce SRIM data by fitting SRIM results with polynomial fits, keeping only the first four moments. We have developed Python routines to model the ion-wall interactions based on these polynomial fits, and since Python is a cross-platform language, these routines work on Linux, Windows, and Mac OS X. Furthermore, by reducing the SRIM data to polynomial fits of only a few coefficients, we have eliminated the need to store large databases (SRIM runs of 10,000 ions can result in $10 \mathrm{~s}$ of $\mathrm{MB}$ of data for each energy and angle).

To generate our reduced description the first step is, for each energy and angle of incident ion, to run SRIM to generate 10,000 results, including whether the ion backscattered or not, and if it backscattered, with what energy and velocities. We then reduce the curves describing the number of scattered ions with a given velocity component as a function of angle for a given incident energy by fitting them with a fourth-order polynomial. Figure 1 shows an example for singly ionized potassium incident on stainless steel. The figure shows these four moment coefficients as a function of incident angle for an incident energy of $2.0 \mathrm{MeV}$ (red curve), along with the actual SRIM results (blue dots). To find a result for an incident energy for which we do not have SRIM data, we interpolate the fourth-order coefficients from the two nearest incident energies.

We show an example in Fig. 2. The blue curves are the reduced, fourth-order fit to the SRIM results for number of scattered ions with a given energy for incident energies of 1.6 and $1.7 \mathrm{MeV}$. The red curve is interpolation for an incident energy of $1.65 \mathrm{MeV}$.
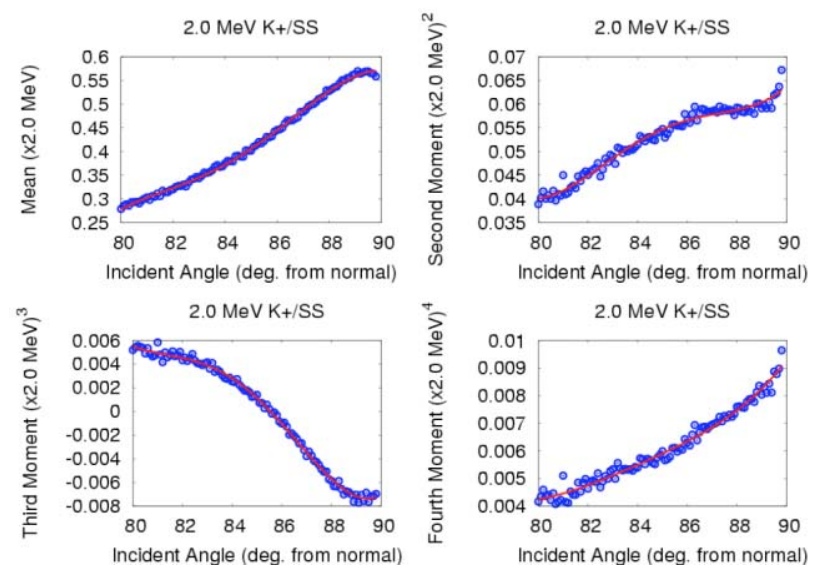

Figure 1: The first four moments describing the reflected energies as a function of incident angle for an incident energy of $2.0 \mathrm{MeV}$. 
Proceedings of 2005 Particle Accelerator Conference, Knoxville, Tennessee

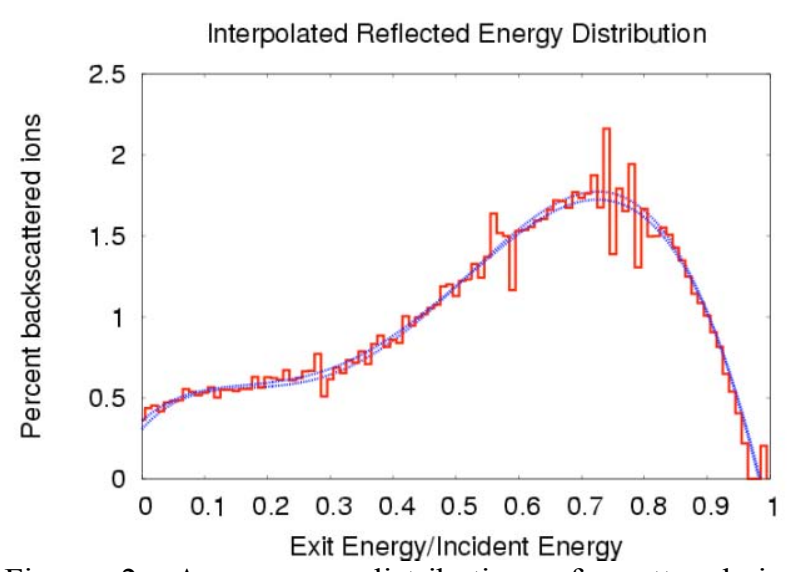

Figure 2: An energy distribution of scattered ions generated using the Python interpolation routines for
SRIM results (red curve). The incident ion was $1.65 \mathrm{MeV}$ singly ionized potassium, incident on stainless steel at 89 degrees to the normal. Also shown are the momentgenerated distributions for $1.7 \mathrm{MeV}$ and $1.8 \mathrm{MeV}$ (blue curves).

\section{REFERENCES}

[1] J. F. Ziegler, J. P. Biersack, and U. Littmark, "The Stopping and Range of Ions in Solids", Pergamon Press, New York (1985).

[2] A. W. Molvik, et. al., Phys. Rev. ST Accel. Beams 7, 093202 (2004) 